\section{Case Reports in Ophthalmology}

\title{
Treatment of Retinitis Pigmentosa- Associated Cystoid Macular Oedema Using Intravitreal Aflibercept (Eylea) despite Minimal Response to Ranibizumab (Lucentis): A Case Report
}

C S. Karger AG, Basel

Provisory PROOF Copy for personal reference only ANY DISTRIBUTION OF THIS ARTICLE WITHOUT WRITTEN CONSENT FROM S. KARGER AG, BASEL IS A VIOLATION OF THE COPYRIGHT.

\section{Stacey A. Strong ${ }^{a}$ Avinash Gurbaxani ${ }^{b}$ Michel Michaelides ${ }^{a, c}$ \\ ${ }^{a}$ Institute of Ophthalmology, University College London, London, UK; ${ }^{b}$ Moorfields Eye Hospital, Dubai, United Arab Emirates; ${ }^{C}$ Moorfields Eye Hospital, London, UK}

\section{Keywords}

Aflibercept · Cystoid macular oedema - Eylea · Retinitis pigmentosa

\begin{abstract}
Background: We present an interesting case of bilateral retinitis pigmentosa (RP)-associated cystoid macular oedema that responded on two separate occasions to intravitreal injections of aflibercept, despite previously demonstrating only minimal response to intravitreal ranibizumab. This unique case would support a trial of intravitreal aflibercept for the treatment of RP-associated cystoid macular oedema. Case Presentation: A 38-year-old man from Dubai, United Arab Emirates, presented to the UK with a 3-year history of bilateral RP-associated cystoid macular oedema. Previous treatment with topical dorzolamide, oral acetazolamide, and intravitreal ranibizumab had demonstrated only minimal reduction of cystoid macular oedema. Following re-confirmation of the diagnosis by clinical examination and optical coherence tomography imaging, bilateral loading doses of intravitreal aflibercept were given. Central macular thickness reduced and the patient returned to Dubai. After 6 months, the patient was treated with intravitreal ranibizumab due to re-accumulation of fluid and the unavailability of aflibercept in Dubai. Only minimal reduction of central macular thickness
\end{abstract}


was observed. Once available in Dubai, intravitreal aflibercept was administered bilaterally with further reduction of central macular thickness observed. Visual acuity remained stable throughout. Conclusions: This is the first case report to demonstrate a reduction of RPassociated $\mathrm{CMO}$ following intravitreal aflibercept, despite inadequate response to ranibizumab on two separate occasions. Aflibercept may provide superior action to other antiVEGF medications due to its intermediate size $(115 \mathrm{kDa})$ and higher binding affinity. This is worthy of further investigation in a large prospective cohort over an extended time to determine the safety and efficacy of intravitreal aflibercept for use in this condition.

\section{Background}

Retinitis pigmentosa (RP) is the most prevalent inherited retinal disease (IRD), with IRD now representing the commonest cause of visual impairment registration in the working age population and the second commonest in childhood in the UK [1]. Typical symptoms of RP include nyctalopia, photopsia, and progressive visual field loss; however, vision can also be affected by cataracts and/or cystoid macular oedema (CMO).

Around $20 \%$ of RP patients develop CMO, the pathogenesis of which is not clearly understood. Suggested mechanisms include: anti-retinal antibodies [2], retinal pigment epithelium dysfunction [3], Muller cell oedema [4], and vitreous traction [5].

Many treatments have been attempted for RP-associated CMO, including grid laser, vitrectomy, oral lutein, intravitreal dexamethasone, intravitreal triamcinolone, topical carbonic anhydrase inhibitors, oral carbonic anhydrase inhibitors, oral corticosteroids, topical non-steroidal anti-inflammatory medication, and topical steroid [6-10]. However, all of the aforementioned treatments have limited and highly variable efficacy. This, together with several side effects of these medications that markedly restrict their use, has led to the search to find alternative therapies that are both well tolerated and are more consistently effective.

Intravitreal anti-vascular endothelial growth factor (anti-VEGF) medication is now licenced for use within the UK for CMO secondary to macular degeneration, diabetic retinopathy, and retinal vein occlusion. While the pathogenesis of RP-associated CMO is still unclear, it is believed that VEGF may play a role in the development of CMO. It has therefore been suggested as an alternative treatment for RP-associated CMO.

Limited data has been published regarding the use of intravitreal anti-VEGF medication for RP-associated CMO. Querques et al. [11] observed improvement of visual acuity and macular thickness in a patient with refractory RP-associated CMO taking oral acetazolamide, 1 month following a single injection of intravitreal pegaptanib (MACUGEN; EyeTech Pharmaceutical, Inc., New York, N.Y., USA). No recurrence of CMO was seen at 4 months postinjection [11]. Melo et al. [12] observed no improvement in 2 patients with RP-associated CMO treated with a single injection of intravitreal bevacizumab (AVASTIN; Genentech, South San Francisco, Calif., USA); however, Yuzbasioglu et al. [13] documented improvement of macular thickness and visual acuity in all 13 eyes of 7 patients.

Artunay et al. [14] treated 15 eyes with RP-associated CMO with intravitreal ranibizumab and compared them with 15 eyes of similar patients who refused treatment. A significant improvement in macular thickness was observed in those patients treated with intravitreal ranibizumab. 
In a recent case report, a single unilateral intravitreal injection of aflibercept was given to a patient with RP-associated CMO. Improvement in both visual acuity and macular thickness was seen at 1 month post-injection as well as maintenance of this improvement documented at 6 months [15]. Aflibercept is a recombinant fusion protein consisting of portions of the extracellular domains of human VEGF receptors 1 and 2 fused to the Fc portion of human IgG1. This unique design is what sets aflibercept apart from other intravitreal antiVEGF medications by enabling its action as a decoy receptor.

\section{Case Presentation}

In August 2013, a 38-year-old man from Dubai, United Arab Emirates, was seen by a medical retina specialist in the UK. He was previously diagnosed with autosomal recessive RP in Dubai and bilateral CMO had been documented over the last 3 years. The patient had undergone uncomplicated bilateral cataract surgery with the insertion of posterior chamber intraocular lenses in 2004. There was no other relevant past medical history. Family history revealed parental consanguinity, with his parents being first cousins.

He had previously received topical dorzolamide and a 3-week trial of oral acetazolamide (Diamox) $250 \mathrm{mg}$ three times per day, with no significant improvement in the degree or extent of CMO. Bilateral injections of ranibizumab (LUCENTIS; Genentech, South San Francisco, Calif., USA) had been performed once a month for 3 months in Dubai in 2013 with only minimal response observed. At the time of the consultation in the UK, the patient was no longer receiving topical or oral treatment for CMO.

On examination, BCVA was $6 / 18$ in the right eye and 6/36 in the left eye. Visual field testing to confrontation revealed constricted fields of 10-20 degrees in both eyes. Fundoscopy revealed bilateral dense bone spicules, bilateral CMO, attenuated retinal vessels, and pale optic discs. Spectral domain optical coherence tomography (SDOCT) showed marked bilateral CMO with central macular thickness (CMT) of 394 and $414 \mu \mathrm{m}$ in the right and left eye, respectively (fig. 1A, E).

The anti-VEGF medication selected for use in this patient was aflibercept (EYLEA; Regeneron Pharmaceuticals, Inc., Tarrytown, New York, N.Y., USA, and Bayer Healthcare Pharmaceuticals, Berlin, Germany). This was due to there being only a minimal response observed following treatment with ranibizumab as well as consideration that its effects may be longer lasting than other anti-VEGF medication. The risks and benefits of treatment with aflibercept together with its off-label use were discussed with the patient. It was also highlighted that there was a limited evidence base for its usage in RP-associated CMO.

Informed consent was taken and bilateral intravitreal injections of $0.05 \mathrm{ml}$ aflibercept ( $40 \mathrm{mg} / \mathrm{ml}$ ) given via standard aseptic technique. There were no peri-operative complications. Post-operative chloramphenicol drops were prescribed.

One month after treatment, BCVA improved to 6/12 in the right eye but remained 6/36 in the left eye. The patient did not notice any subjective improvement. SDOCT revealed markedly less CMO in both eyes, with CMT of 263 and $243 \mu \mathrm{m}$ in the right and left eye, respectively (fig. 1B, F). A second uncomplicated intravitreal injection of aflibercept was undertaken bilaterally.

One month after his second injection of aflibercept, BCVA remained at 6/12 in the right eye and 6/36 in the left eye. At this visit, the patient reported a subjective improvement of vision. SDOCT revealed a similar level of CMO bilaterally compared to the previous visit. CMT was 268 and $239 \mu \mathrm{m}$ in the right and left eye, respectively (fig. 1C, G). 
The patient then returned to the United Arab Emirates where he was seen by a medical retina specialist in Dubai, in December 2013. BCVA was 6/18 in the right eye and 6/24 in the left eye. SDOCT revealed a similar level of CMO bilaterally (fig. 1D, H), despite his last aflibercept injection being 8 weeks prior. CMT was recorded as 253 and $224 \mu \mathrm{m}$ in the right and left eye, respectively. As the patient was stable, the decision was taken not to treat with an alternative anti-VEGF, since aflibercept was due to be made available for use in Dubai from January 2014.

Unfortunately, there was an unexpected delay in aflibercept being made available for use in Dubai. In March 2014 (5 months after the patient's last injection of aflibercept), BCVA was 6/18 in the right eye and 6/36 in the left eye. SDOCT revealed a significant increase of CMO bilaterally, with CMT of 385 and $434 \mu \mathrm{m}$ in the right and left eye, respectively (fig. $2 \mathrm{~A}$, C). In order not to delay treatment any further, the decision was taken to perform 3 monthly loading doses of ranibizumab bilaterally. The response to ranibizumab was markedly less pronounced compared to aflibercept. In May 2014, BCVA remained at 6/18 in the right eye and had decreased to 6/48 in the left eye. SDOCT revealed bilateral CMO, with CMT of 304 and $342 \mu \mathrm{m}$ in the right and left eye, respectively (fig. 2B, D). The decision was taken not to perform further injections of ranibizumab.

In August 2014, the patient had an increased amount of CMO. SDOCT measured 452 and $513 \mu \mathrm{m}$ in the right and left eye, respectively (fig. 3A, E). Fortunately, aflibercept became available for use in Dubai, and the patient received treatment with 3 monthly loading doses of aflibercept bilaterally. A good response was noted once again. In October 2014, BCVA had improved to $6 / 18$ in the right eye and 6/36 in the left eye. SDOCT showed a marked reduction in CMO (fig. 3B, F), measuring 248 and $226 \mu \mathrm{m}$ in the right and left eye, respectively.

The patient continued to receive aflibercept injections in January 2015, March 2015, June 2015, and September 2015 and CMT remained stable (fig. 3C, G). In September 2015, BCVA was $6 / 15$ in the right eye, 6/36 in the left eye, with stable CMT of 250 and $194 \mu \mathrm{m}$ in the right and left eye, respectively (fig. 3D, H). Figure 4 summarises the effect of anti-VEGF medications on CMT over time.

\section{Conclusions}

There are currently no proven treatments for RP-associated CMO. The use of medication, such as carbonic anhydrase inhibitors, has demonstrated inconsistent efficacy and unwanted side effects. FDA approval of anti-VEGF medication has now extended to include CMO secondary to age-related macular degeneration, diabetic retinopathy, and retinal vein occlusion. While the pathogenesis of RP-associated CMO is not entirely understood, VEGF may play a role in the formation of RP-associated CMO thereby representing a potential target for treatment. RP is not an ischaemic condition and if anything, the natural bone spicule formation secondary to photoreceptor cell death results in an overall reduction of oxygen consumption by the retina. We hypothesise that a localised source of VEGF produced, for example, by Muller cells under pathological conditions contributes to CMO formation whilst also explaining why it is rare to find reports of peripheral neovascularisation in RP.

This is the first case report to demonstrate a reduction of RP-associated CMO following intravitreal aflibercept, despite inadequate response to ranibizumab. Aflibercept may provide superior action to other anti-VEGF medications due to its intermediate size (115 kDa) and higher binding affinity. This is worthy of further investigation in a large prospective 
cohort over an extended time to determine the safety and efficacy of aflibercept for use in this condition.

\section{Statement of Ethics}

The patient has given his informed consent for this case report to be written.

\section{Disclosure Statement}

Funding was not received to write this case report; however, Bayer Plc will be approached to cover the publication costs should this article be accepted.

The authors S.A.S. and M.M. declare that they are currently being funded by Bayer Plc. to set up a phase II exploratory trial at Moorfields Eye Hospital, London, UK, to review the safety and efficacy of aflibercept for RP-associated CMO.

\section{References}

1 Liew G, Michaelides M, Bunce C: A comparison of the causes of blindness certifications in England and Wales in working age adults (16-64 years), 1999-2000 with 2009-2010. BMJ Open 2014;4:e004015.

2 Heckenlively JR, Jordan BL, Aptsiauri N: Association of antiretinal antibodies and cystoid macular edema in patients with retinitis pigmentosa. Am J Ophthalmol 1999;127:565-573.

3 Strauss 0: The retinal pigment epithelium in visual function. Physiological reviews 2005;85:845-881.

4 Reichenbach A, Wurm A, Pannicke T, Iandiev I, Wiedemann P, Bringmann A: Muller cells as players in retinal degeneration and edema. Graefes Arch Clin Exp Ophthalmol 2007;245:627-636.

5 Schepens CL, Avila MP, Jalkh AE, Trempe CL: Role of the vitreous in cystoid macular edema. Surv Ophthalmol 1984;28(suppl):499-504.

6 Liew G, Moore AT, Webster AR, Michaelides M: Efficacy and prognostic factors of response to carbonic anhydrase inhibitors in management of cystoid macular edema in retinitis pigmentosa. Invest Ophthalmol Vis Sci 2015;56:1531-1536.

7 Adackapara CA, Sunness JS, Dibernardo CW, Melia BM, Dagnelie G: Prevalence of cystoid macular edema and stability in OCT retinal thickness in eyes with retinitis pigmentosa during a 48-week lutein trial. Retina 2008;28:103-110.

8 Garcia-Arumi J, Martinez V, Sararols L, Corcostegui B: Vitreoretinal surgery for cystoid macular edema associated with retinitis pigmentosa. Ophthalmology 2003;110:1164-1169.

9 Newsome DA, Blacharski PA: Grid photocoagulation for macular edema in patients with retinitis pigmentosa. Am J Ophthalmol 1987;103:161-166.

10 Barge S, Rothwell R, Sepulveda P, Agrelos L: Intravitreal and subtenon depot triamcinolone as treatment of retinitis pigmentosa associated cystoid macular edema. Case Rep Ophthalmol Med 2013;2013:591681.

11 Querques G, Prascina F, Iaculli C, Noci ND: Intravitreal pegaptanib sodium (Macugen) for refractory cystoid macular edema in pericentral retinitis pigmentosa. Int Ophthalmol 2009;29:103-107.

12 Melo GB, Farah ME, Aggio FB: Intravitreal injection of bevacizumab for cystoid macular edema in retinitis pigmentosa. Acta Ophthalmol Scand 2007;85:461-463.

13 Yuzbasioglu E, Artunay O, Rasier R, Sengul A, Bahcecioglu H: Intravitreal bevacizumab (Avastin) injection in retinitis pigmentosa. Curr Eye Res 2009;34:231-237.

14 Artunay O, Yuzbasioglu E, Rasier R, Sengul A, Bahcecioglu H: Intravitreal ranibizumab in the treatment of cystoid macular edema associated with retinitis pigmentosa. J Ocul Pharmacol Ther 2009;25:545550 .

15 Moustafa GA, Moschos MM: Intravitreal aflibercept (Eylea) injection for cystoid macular edema secondary to retinitis pigmentosa - a first case report and short review of the literature. BMC Ophthalmol 2015;15:44. 


\section{Case Reports in Ophthalmology}

Case Rep Ophthalmol 2016;7:389-397

DOI: 10.1159/000448427

c) 2016 The Author(s). Published by S. Karger AG, Basel www.karger.com/cop

Strong et al: Treatment of Retinitis Pigmentosa-Associated Cystoid Macular Oedema Using Intravitreal Aflibercept (Eylea) despite Minimal Response to Ranibizumab (Lucentis): A Case Report

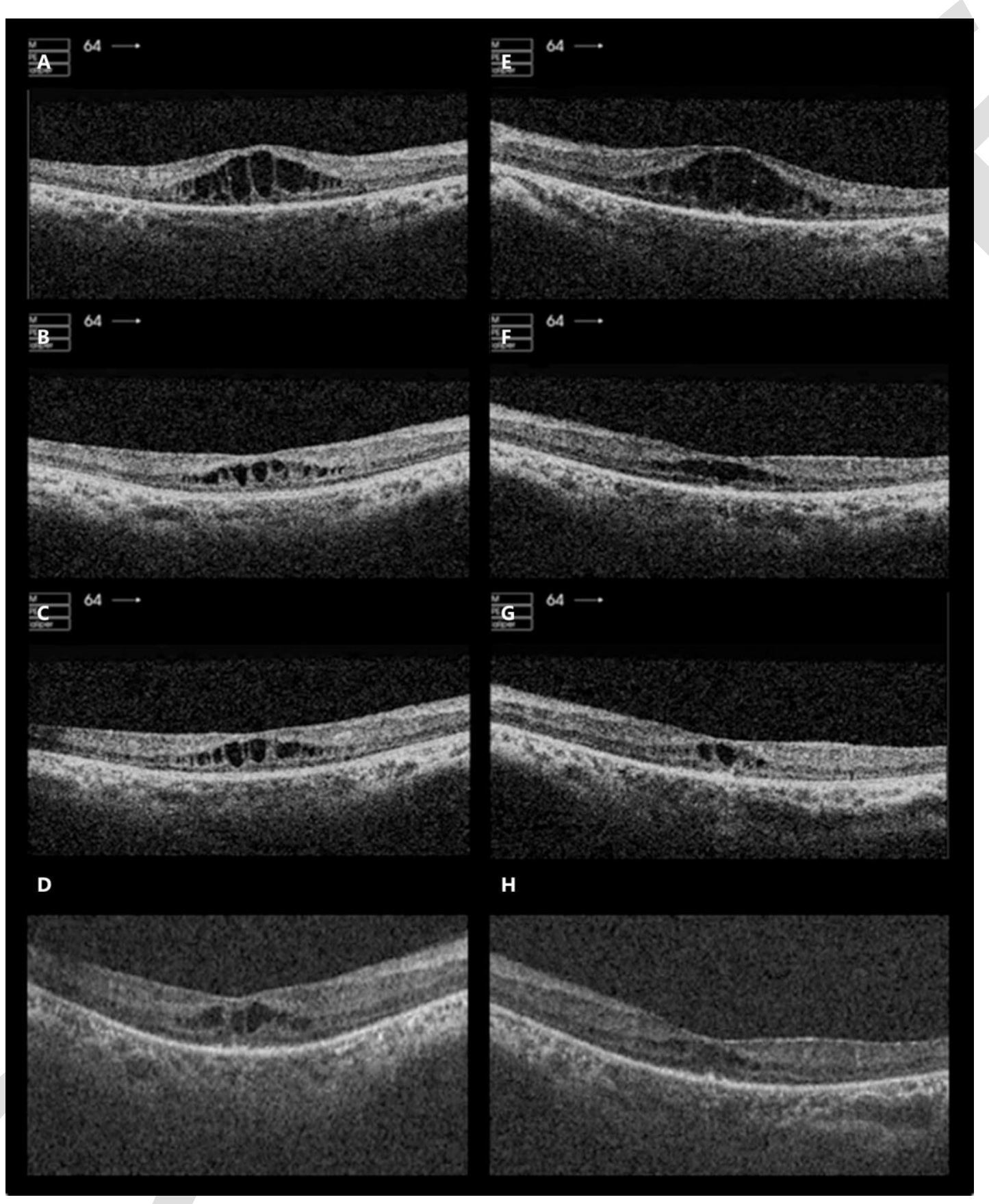

Fig. 1. OCT of both eyes before and after intravitreal injections of aflibercept given in the UK. OCT in the right eye before injection of aflibercept (A), 1 month after the first injection of aflibercept (B), 1 month after the second injection of aflibercept (C), and 8 weeks after the third injection of aflibercept (D). OCT in the left eye before injection of aflibercept (E), 1 month after the first injection of aflibercept (F), 1 month after the second injection of aflibercept $(\mathbf{G})$, and 8 weeks after the third injection of aflibercept $(\mathbf{H})$. 


\section{Case Reports in Ophthalmology}

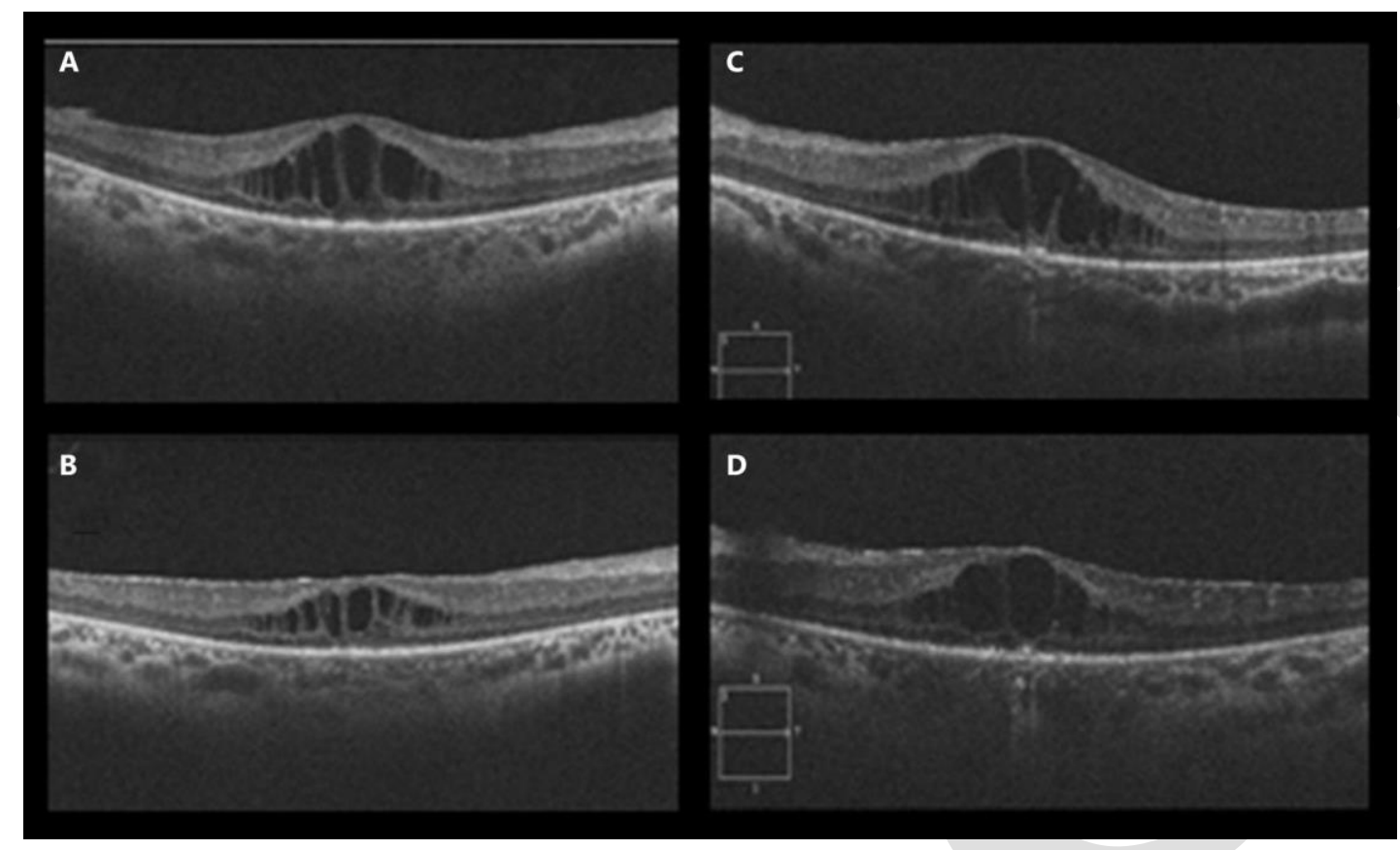

Fig. 2. OCT of both eyes before and after intravitreal injections of ranibizumab given in Dubai. OCT in the right eye immediately before injection of ranibizumab $(\mathbf{A})$ and on the day he received his third injection of ranibizumab (C). OCT in the left eye immediately before injection of ranibizumab (B) and on the day he received his third injection of ranibizumab (D).

Strong et al: Treatment of Retinitis Pigmentosa-Associated Cystoid Macular Oedema Using Intravitreal Aflibercept (Eylea) despite Minimal Response to Ranibizumab (Lucentis): A Case Report 


\section{Case Reports in Ophthalmology}

\begin{tabular}{l|l}
\hline Case Rep Ophthalmol 2016;7:389-397 \\
\hline DOI: 10.1159/000448427 & $\begin{array}{l}\text { @ 2016 The Author(s). Published by S. Karger AG, Basel } \\
\text { www.karger.com/cop }\end{array}$ \\
\hline
\end{tabular}

Strong et al: Treatment of Retinitis Pigmentosa-Associated Cystoid Macular Oedema Using Intravitreal Aflibercept (Eylea) despite Minimal Response to Ranibizumab (Lucentis): A Case Report
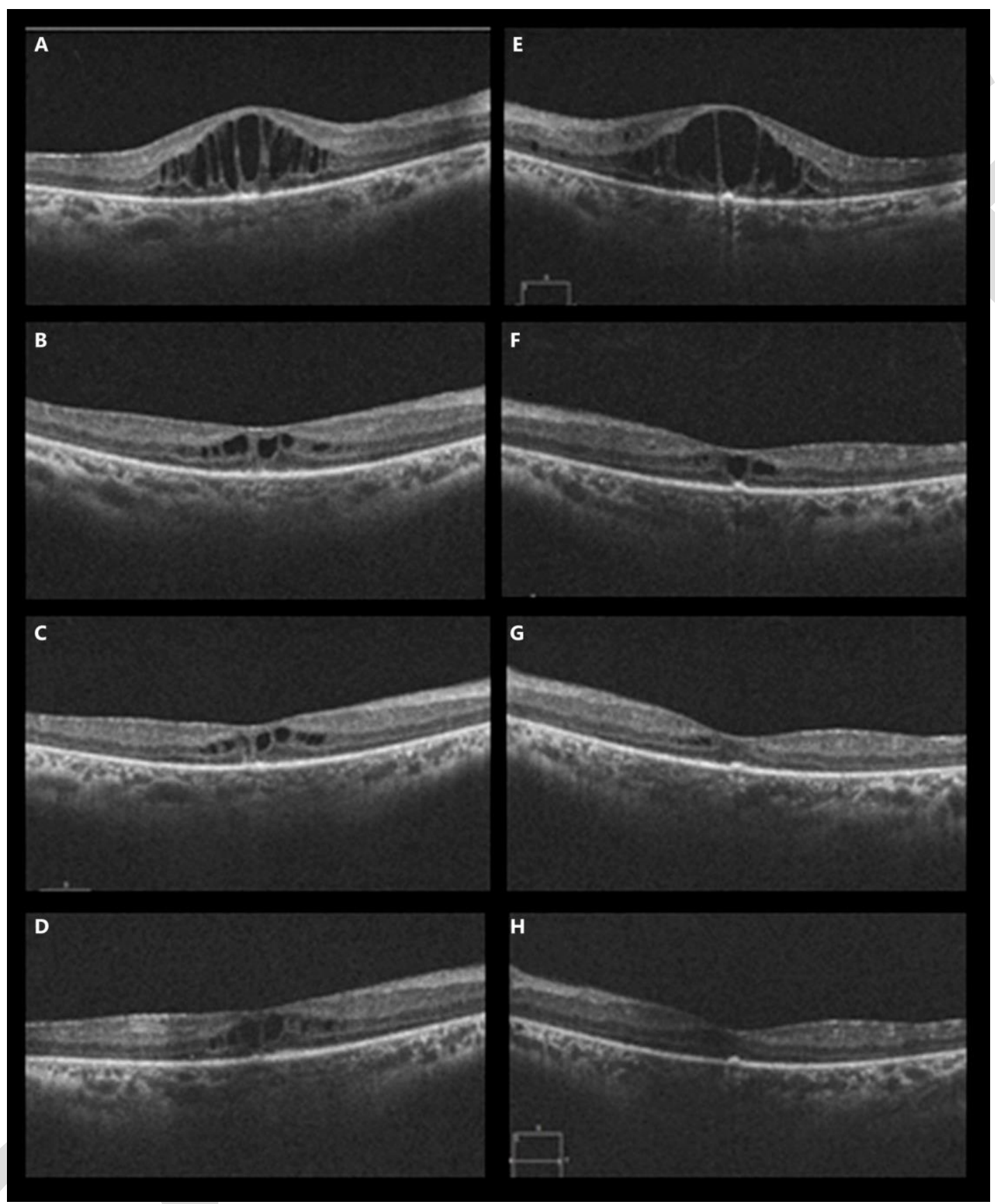

Fig. 3. OCT of both eyes before and after intravitreal injections of aflibercept given in Dubai. OCT in the right eye immediately before injection of aflibercept (A), immediately before the third injection of aflibercept (B), 2 months following the fourth injection of aflibercept (C), and 3 months following the sixth injection of aflibercept (D). OCT in the left eye immediately before injection of aflibercept (E), immediately before the third injection of aflibercept (F), 2 months following the fourth injection of aflibercept (G), and 3 months following the sixth injection of aflibercept $(\mathbf{H})$. 
A graph to demonstrate change in central macular thickness (CMT) over time

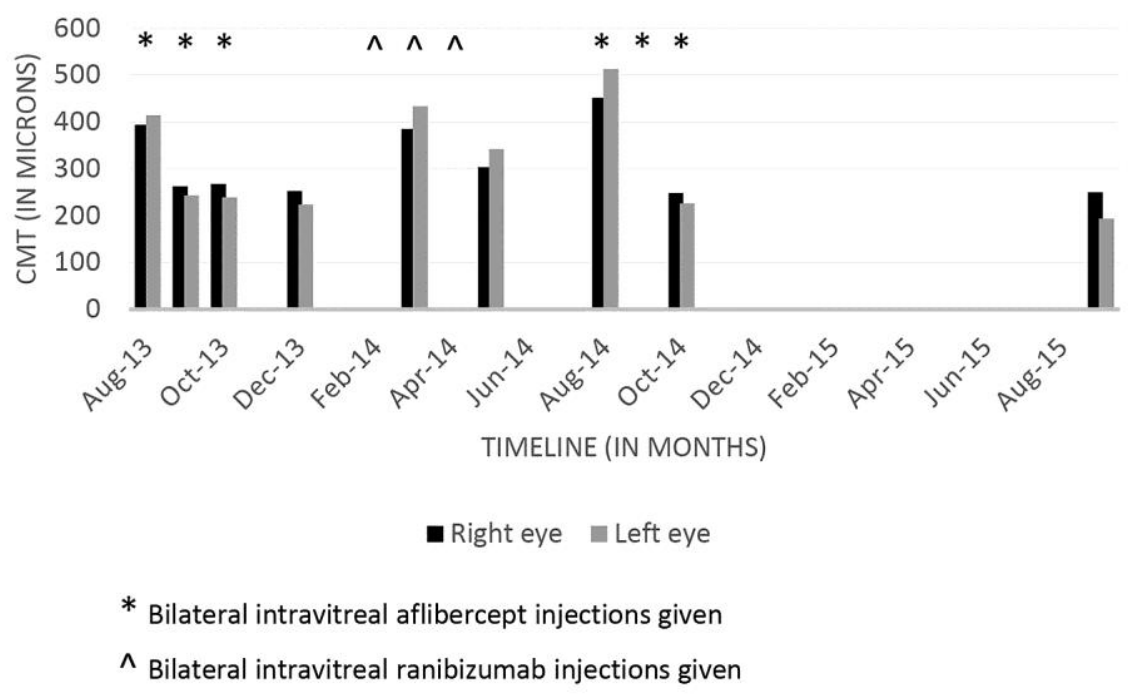

Fig. 4. The effect of anti-VEGF medications on CMT over time is presented. 